\title{
The Effect of Bacterial Adhesion on Grafted Chains Revealed by the Non-Invasive Sum Frequency Generation Spectroscopy
}

\author{
Emilie Bulard, ${ }^{1}$ Marie-Pierre Fontaine-Aupart, ${ }^{1}$ Henri Dubost, ${ }^{1}$ \\ Wanquan Zheng, ${ }^{1}$ Jean-Marie Herry, ${ }^{2}$ Marie-Noëlle Bellon-Fontaine, ${ }^{2}$ \\ Romain Briandet, ${ }^{2}$ and Bernard Bourguignon ${ }^{1}$ \\ ${ }^{1}$ Institut des Sciences Moléculaires d Orsay, ISMO-CNRS, Université Paris Sud 11, \\ 91405 Orsay, France \\ ${ }^{2}$ INRA AgroParisTech, UMR 1319, Micalis, 91300 Massy, France
}

Correspondence should be addressed to Emilie Bulard, emilie.bulard@u-psud.fr

Copyright (C) 2012 Emilie Bulard et al. This is an open access article distributed under the Creative Commons Attribution License, which permits unrestricted use, distribution, and reproduction in any medium, provided the original work is properly cited.

\begin{abstract}
In biomedical and food industry, surface colonization by bacteria is harmful: it leads to biofilm formation, a microbial consortia more resistant to antibiotics than planktonic bacteria. In order to design materials able to limit the biofilm formation, the effect of bacteria on materials has to be well characterized. In this work, a well-defined surface composed of a self-assembled monolayer (SAM) of octadecanethiol (ODT) onto a gold surface is probed in situ. The SAM conformation is obtained using the femtosecond vibrational sum frequency generation (SFG) spectroscopy. This technique provides selectively the molecular vibrational signature of the interface. The behaviour of the ODT SAM is studied in different environments: in air, in water, and upon exposure to hydrophilic or hydrophobic Lactococcus lactis bacteria. Modelling the experimental SFG spectra reveals a measurable change of the SAM conformation which depends on the environment, especially on the hydrophilic-hydrophobic character.
\end{abstract}

Keywords: Bacterial adhesion, SFG spectroscopy, surface, self-assembled monolayers, molecular conformation

\section{Introduction}

All surfaces are exposed to living entities such as bacteria in natural environments. The initial colonization of the surface by pathogenic bacteria is of concern in many fields such as biomedical or food industry $[1,2]$. Indeed, it evolves into the formation of a biofilm, a three-dimensional bacterial organization where bacteria are more resistant to antibiotics and biocides than planktonic counterparts, leading to nosocomial or food-born infections. Therefore, the scientific community is mobilized to understand the mechanisms involved in the bioadhesive process in order to inhibit and/or control it and find new preventive routes for biofilms control.

Bacteria adhere to an inert surface through noncovalent molecular interactions such as van der Waals, electrostatic, Lewis acid/base interactions, depending on the characteristics of the bacteria (peptidoglycan nature, cell wall proteins and polysaccharides, extracellular appendices as pili, etc.) and 
the physicochemical properties of the surface [3, 4]. Experiments have been carried out to understand bacterial adhesion on functionalized polymers or grafted chains, by changing the topography, the hydrophobic/hydrophilic character of the surface, and so forth [5, 6]. However, divergences appear between predictive models and experimental results [7, 8], likely due to the lack of non-invasive surface characterization at the molecular level when bacteria are on the surface.

The aim of the present study is to characterize the initial event of the bacterial colonization with a method able to provide information on the surface at the molecular level and in situ. The vibrational sum frequency generation (SFG) spectroscopy is employed here for the first time to probe a specific surface: a self-assembled monolayer (SAM) of octadecanethiol (ODT) onto a gold surface colonized by bacteria. This technique, based on second-order nonlinear optics, consists in overlapping in time and in space an infrared (tuned in this case to $\mathrm{C}-\mathrm{H}$ band wavelengths) and a visible beam. The main interest of this spectroscopy is that the SFG response originates only from noncentrosymmetric regions (in general, the bulk of solids does not contribute to the SFG signal) allowing to acquire specifically the molecular spectral signature of the surface which can be related to its molecular conformation. SFG spectroscopy appears as a nondestructive method, well adapted to study selectively interfaces of biomolecular systems [9-11] due to its high sensitivity. Previous SFG studies have described the conformation of ODT SAM exposed to air [12]. In this work, the conformation of ODT SAM is determined when this surface is exposed to pure water and to an aqueous solution of hydrophilic or hydrophobic Lactococcus lactis cells (nonpathogenic models for Streptococcus agalactie responsible of harsh neonatal infections).

It is shown that the spectral signature of the SAM in contact with bacteria can be detected using femtosecond IR-visible sum frequency generation spectroscopy. Moreover, modelling the experimental SFG spectra reveals a measurable change of the SAM conformation depending on the hydrophobichydrophilic character of the environment. These results show that bacteria are able to modify their support at the molecular level [13]. Therefore, this result should be taken into account for the design of new biomaterials.

\section{Materials and Methods}

\subsection{Material}

Substrates consisted of borosilicate glass coated with a polycrystalline gold film of $250 \mathrm{~nm}$ thickness and annealed in an oven at $600^{\circ} \mathrm{C}$ during 30 seconds. A self-assembled monolayer of octadecanethiol was created on the gold-coated substrate employing the following procedure: the coated surface was dipped in a $1 \mathrm{mM}$ ODT solution in absolute ethanol during 3 hours, rinsed in absolute ethanol and dried under nitrogen flow. As described previously [12], we obtained in these conditions an ODT SAM thickness of $\sim 2 \mathrm{~nm}$. Surface topography of these substrates was characterized by atomic force microscopy (AFM) in contact mode [13]. The gold surface topography presents plantens of $1-2 \mu \mathrm{m}^{2}$ delimitated by grooves of 20-30 nm depth. Moreover, water contact angle measurements were performed: the ODT SAM presents a contact angle of $109 \pm 2^{\circ}$ on all its surface so a strong hydrophobic character.

\subsection{Lactococcus lactis Bacteria}

Bacteria used for the adhesion on ODT SAMs were L. lactis ssp. cremoris strains MG1363 and its derivative mutant PRTP+ expressing the PrtP protease $[14,15]$. Bacteria were stored at $-20^{\circ} \mathrm{C}$ in 
M17 broth (Difco) containing $0.5 \%$ (w,vol) of glucose and 50\% (vol/vol) of glycerol. They were subcultured twice in M17-glucose broth at $30^{\circ} \mathrm{C}$, until stationary phase was reached. Finally, they were cultivated overnight (working cultures) at $30^{\circ} \mathrm{C}$. Also, $90 \mathrm{~mL}$ of bacteria from final working cultures were harvested by centrifugation $\left(10\right.$ minutes, $\left.7000 \mathrm{~g}, 4^{\circ} \mathrm{C}\right)$, washed twice, and resuspended in $25 \mathrm{~mL}$ of distilled water at a final cell density of approximately $4.10^{9}$ cells $/ \mathrm{mL}$.

The microbial adhesion to solvents (MATSs) method was employed for the evaluation of the hydrophobic/hydrophilic character of the cell surface of the two strains. The wild-type L. lactis strain MG1363 presented a weak affinity $(7 \pm 5 \%)$ to both hydrophobic decane and hexadecane indicating a hydrophilic cell wall, whereas cells from the strains MG1363 PRTP+ presented a strong affinity of $(90 \pm 5) \%$ indicating their strong hydrophobic character.

\subsection{Bacterial Adhesion}

To investigate the ODT SAM response to bacterial adhesion, a volume of $\sim 200 \mu \mathrm{L}$ of the bacterial suspension at $10^{9}$ cells $/ \mathrm{mL}$ in distilled water was deposited over the ODT SAM. The solution was allowed to incubate for $90 \mathrm{~min}$ and then washed with the suspending fluid to remove the non-adherent

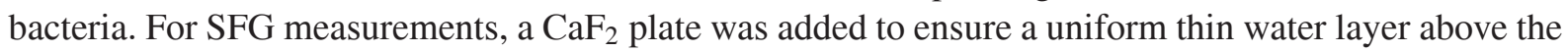
surface sample. Its thickness was $\sim 5 \mu \mathrm{m}$, which is sufficiently thin to allow infrared radiation to reach the sample.

To control in situ the bacterial surface coverage on the ODT SAM, scanning electron microscopy (SEM, described previously [13]) and epifluorescence microscopy measurements were performed. For epifluorescence images, adherent bacteria were stained with the nucleic acid dye acridin orange $(0.01 \%$ in water) for 15 minutes in the dark. The dye solution was washed and replaced by pure water before mounting the sample under a Leica DM2 microscope equipped with an Olympus Camedia C5060WZ digital camera. Both microscopies show that our procedure ensures a homogeneous bacterial deposit. The bacterial surface coverage was estimated to $50 \pm 20 \%$ of the ODT SAM surface, corresponding to 2200 ODT molecules across the diameter of each bacterium.

Morphology of adherent bacteria on ODT SAM was also analyzed by SEM. Lactococcus lactis cells maintain their characteristic ovococcoid morphology after adhesion on the functionalized gold surface. There is no evidence in our collection of SEM images that bacteria are preferentially localized on the grooves of the film. Moreover, no extracellular matrix is present on the surface demonstrating that experimental conditions allow us to stay in the first bacterial colonization step.

\subsection{SFG Technique}

Details about our broad-band SFG setup can be found in our previously published work [12]. Tunable IR pulses ( $4 \mu \mathrm{J}, 145 \mathrm{fs}$ and $150 \mathrm{~cm}^{-1}$ bandwidth) and "visible" pulses ( $800 \mathrm{~nm}, 2 \mu \mathrm{J}$, adjustable duration and bandwidth $1-6 \mathrm{ps} \equiv 15-2.5 \mathrm{~cm}^{-1}$ ) are superimposed on the sample in a collinear copropagating configuration at the incident angle of $\sim 66^{\circ}$ in p polarization. In this experimental geometry, the laser spot size on the sample surface is $\sim 100 \mu \mathrm{m}$. The generated SFG signals are collected during 100 to 300 seconds to obtain an acceptable signal to noise ratio and analyzed by a high-resolution detection system (a spectrometer of resolution $0.4 \mathrm{~cm}^{-1}$ at $650 \mathrm{~nm}$, equipped with a cooled CCD camera). The vibrational 
bands are superimposed to a so-called nonresonant background which has the spectral profile of the IR laser and arises from the broad resonant response of the gold surface electronic states. In order to deconvolute the vibrational bands from this nonresonant background, experimental spectra are fitted to the standard formula:

$$
I\left(\omega_{\mathrm{SFG}}\right) \propto g\left(\omega_{\mathrm{IR}}\right)\left|\chi_{\mathrm{NR}} \cdot e^{i \varphi}+\sum_{v} \frac{A v}{\omega_{\mathrm{IR}}-\omega_{v}+i \Gamma}\right|^{2}
$$

where $g\left(\omega_{\mathrm{IR}}\right)$ is the IR laser spectral profile recorded on a GaAs reference sample which provides only a nonresonant SFG signal. The term $\chi_{\mathrm{NR}} \cdot e^{i \varphi}$ is the Au constant nonresonant response with phase $\varphi$ and $A_{\nu}, \omega_{\nu}$, and $\Gamma$ are the Lorentzian amplitude, frequency, and half width of mode $\nu$, respectively. $\Gamma$ is related to the decay of vibrational energy to the surface and it is supposed to be the same for all $\mathrm{CH}$ modes. The reference GaAs spectrum is measured in air. Therefore, the IR laser profile $g\left(\omega_{\text {IR }}\right)$ must be corrected from absorption by the water layer that surrounds the bacteria. In our fitting procedure, the well-known $\mathrm{CH}$ stretch frequencies are fixed and the width of each stretching mode is the same for all bands. The value of the phase in the different media is also fixed and corresponds to an average obtained over $\sim$ fifty experiments. This limitation of the number of fitted parameters ensures that we cannot obtain multiple solutions [16]. We only admit a phase change from air to water, because the first water layers may affect electrostatically the Au substrate.

\section{Results}

\subsection{ODT SAM in Air}

The SFG spectrum in the wavenumber range $2800-3050 \mathrm{~cm}^{-1}$ of the ODT SAM has been reported several times in the literature (spectrum not shown). We have analysed it in details [13] and extracted the two ODT molecular conformations involved in the SAM. The spectrum consists mainly of the three strong bands of the methyl group: the methyl symmetric stretch band at $2875 \mathrm{~cm}^{-1}$, the Fermi resonance of the $\mathrm{CH}_{3}$ symmetric mode with two quantas of the $\mathrm{CH}_{3}$ bending mode at $2936 \mathrm{~cm}^{-1}$, and the methyl asymmetric stretch band at $2964 \mathrm{~cm}^{-1}$. The regular $\mathrm{CH}_{2}$ band intensities are $2850 \mathrm{~cm}^{-1}$ (symmetric stretching mode) and $2918 \mathrm{~cm}^{-1}$ (asymmetric one). The $-\mathrm{CH}_{2}-\mathrm{S}$-band frequencies are shifted to higher frequencies (2905 and $2973 \mathrm{~cm}^{-1}$ ) and very weak.

\subsection{ODT SAM in Distilled Water}

The SFG spectrum of ODT SAM in distilled water is displayed in Figure 1(a). Figure 1(b) shows the deconvoluted vibrational bands of the $\mathrm{CH}_{3}$ and $\mathrm{CH}_{2}$ groups according to (2.1). The methyl band intensities are different in water and in air: the relative intensity of the $\mathrm{CH}_{3}$ asymmetric mode is smaller in water than in air. 


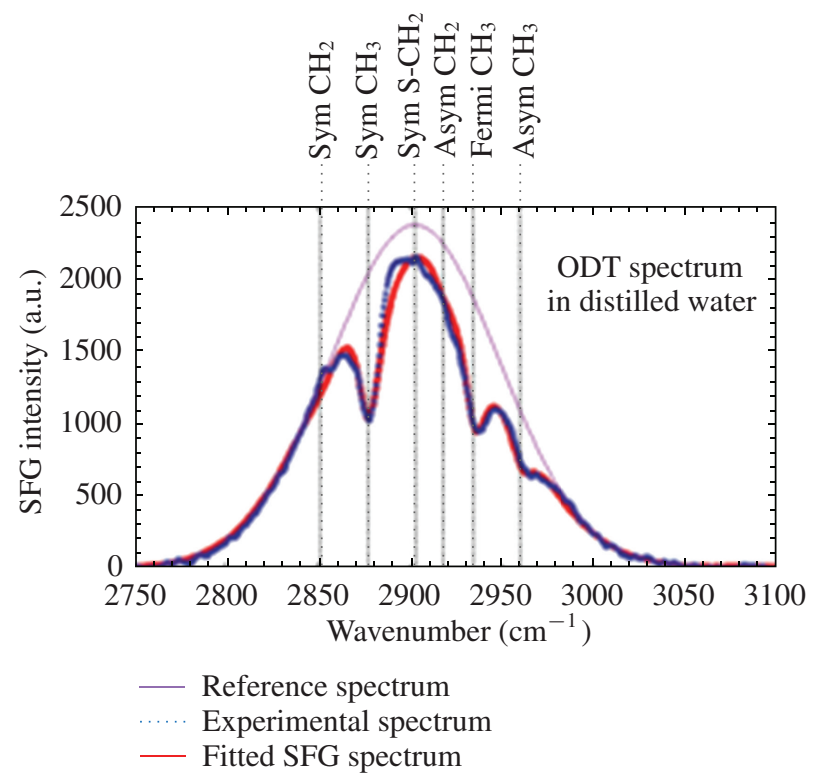

(a)
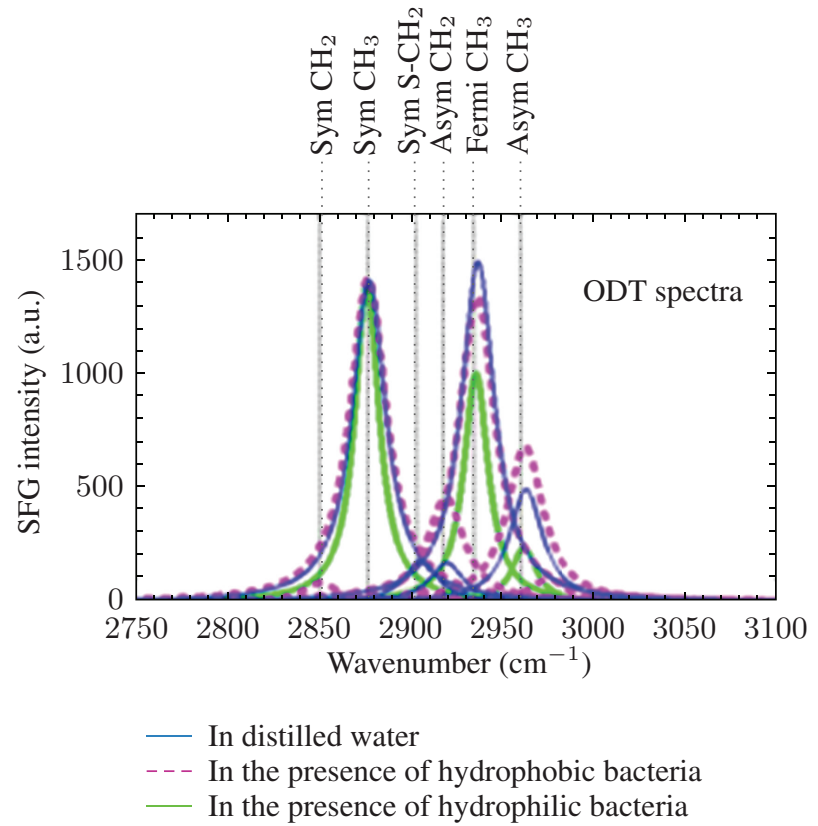

(b)

Figure 1: (a) Experimental (blue dots) and fitted (red lines) SFG spectrum of an ODT SAM in distilled water. The SFG reference spectrum (purple dashed line) is also presented. (b) Deconvoluted vibrational bands of an ODT SAM in distilled water (lines), in the presence of hydrophobic L. lactis bacteria PRTP+ (pink dashed lines), and in the presence of hydrophilic L. lactis bacteria (green lines) in distilled water after 90 minutes of adhesion. 
In order to quantify the intensity changes of the ODT SAM methyl bands, we have defined the phenomenological parameter $R$ equal to the intensity ratio between the $\mathrm{CH}_{3}$ symmetric and the $\mathrm{CH}_{3}$ asymmetric vibrations:

$$
R=\left(I_{\text {sym }}+I_{\text {Fermi }}\right) / I_{\text {asym }}
$$

where the intensity of vibration $i$ is equal to $I_{i}=\left(A_{i} / \Gamma\right)^{2}$. The parameter $R$ measured for ODT in air is $R=3.7 \pm 0.5$ as compared to $R=5.8 \pm 0.5$ when the ODT SAM is exposed to water.

\subsection{ODT SAM in Contact with Hydrophilic and Hydrophobic Bacteria}

We have investigated the effect of bacterial adhesion onto the ODT SAM. For hydrophobic and hydrophilic bacteria, the pattern of the ODT SAM SFG spectra changes compared to that in water (spectra not shown). For both strains, the experimental spectra are fitted to our model as a function of bacterial coverage ranging from 0 to $100 \%$. The best fits are obtained for a bacterial coverage of $\sim 60 \%$ which is in good agreement with coverage estimates derived from fluorescence and SEM images. The relative intensities of vibrational modes and thus the $R$ values differ significantly from those found in water (Figure 1(b)). Moreover, the $R$ value depends on the hydrophilic ( $R=8.8 \pm 0.5)$ /hydrophobic $(R=3.8 \pm 0.5)$ character of the bacteria.

\section{Discussion}

The conformational changes of ODT molecules in the SAM can be extracted from the experimental relative intensities. Owing the fact that interactions between the SAM, water, and bacteria are weak, we assume that molecules are only adjusted by rotation around the axis of their carbon chain. Such a rotation changes the orientation of the methyl groups, which is easily detected by SFG. The intensity of a vibrational mode depends on the orientation of its dipole moment with respect to the surface normal. On metals, only the projection of the transition moment along the surface normal contributes significantly to SFG. Therefore, the $\mathrm{CH}_{3}$ symmetric stretch is strong when the methyl group symmetry axis is perpendicular to the surface. By contrast, the transition moments of the doubly degenerate asymmetric stretching modes are perpendicular to the $\mathrm{CH}_{3}$ symmetry axis and are, therefore, minimal when this axis is perpendicular to the surface. It results that the value of $R$ increases when the methyl terminal part of the ODT SAM raises.

To evaluate quantitatively the methyl orientation of ODT, SFG spectrum modelling of the ODT chains has been performed according to the model described by Bourguignon et al. [12]. The model can calculate the SFG spectrum of adsorbed alkanethiol molecules for any conformation of the alkyl chain. It is based on ab initio calculations of the molecular hyperpolarizability tensors of the modes of individual methyl and methylene groups, and it takes into account the molecular conformation as well as the geometrical and optical parameters such as incidence angles, polarization of the beams, and optical indexes. Moreover, it was established that the ODT SAM structure involves two types of molecules, subscripted $\mathrm{A}$ and $\mathrm{B}$, which differ by the rotation of their $\mathrm{C}$ plane about their average molecular axis. 


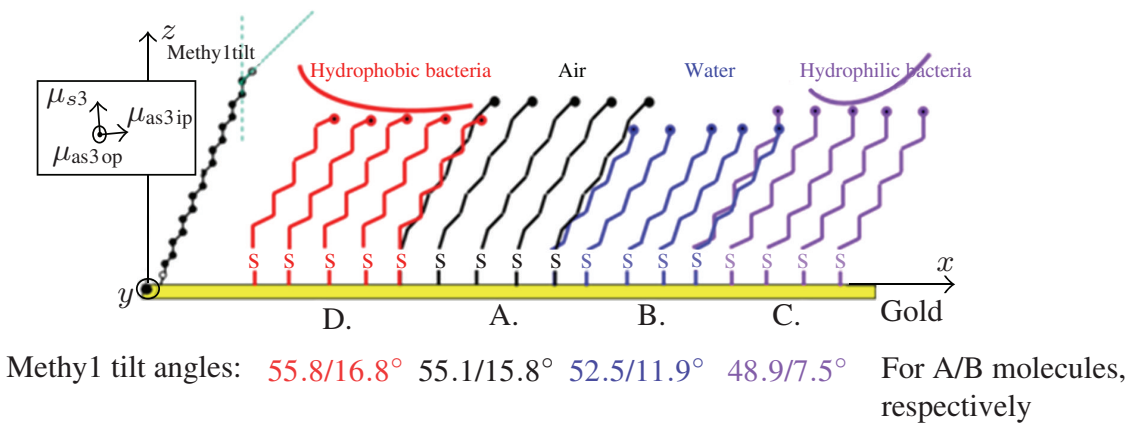

Figure 2: Schematic ODT SAM conformations (the effect of ODT environment is strongly exaggerated for clarity). A: in air. B: in distilled water. C and D show the two extreme possibilities of methyl orientation adjustment allowed by the rotation of the $\mathrm{C}$ backbone planes about the average molecular axis. $\mathrm{C}$ and $\mathrm{D}$ correspond ideally to hydrophilic and hydrophobic interactions, respectively. The directions of the dipole moments of $\mathrm{CH}_{3}$ (symmetric $\mu_{\mathrm{s} 3}$, asymmetric in plane $\mu_{\text {as3ip }}$ and out of plane $\left.\mu_{\text {as3op }}\right)$ are also indicated. Values of methyl axis tilt angle of the ODT SAM in different environments are added below.

Taking into account the data in the SFG spectrum modelling, the methyl axis tilt angle of the ODT SAM is obtained (Figure 2).

When exposed to air, the methyl axis tilt angle is $55.1 / 15.8^{\circ}$ for molecules $\mathrm{A}$ and $\mathrm{B}$, respectively (Figure 2(A)).

Methyl SFG intensities happen to be extremely sensitive to the methyl axis tilt angle: a change of $5^{\circ}$ results in the doubling of the $R$ parameter. We expect, therefore, that SFG will allow probing conformational changes of the SAM even in the case where they are due to weak interactions. When exposed to distilled water, the tilt of the $\mathrm{CH}_{3}$ axis decreases slightly to the value of 1.7 and $2.5^{\circ}$ for molecules A and B with respect to air, respectively: the aqueous environment has a "brush effect" on ODT (Figure 2(B)).

In the case of bacterial adhesion, the spectral changes and so the $R$ value changes could result from a change of the ODT SAM conformation, and/or from an additional signal from the bacteria themselves. We have recorded the SFG spectrum after bacterial adhesion on a bare gold surface (spectrum not shown): only a nonresonant signal is obtained, indicating that bacteria do not contribute to the SFG spectrum and that the observed relative intensity changes result only from a change of the ODT SAM conformation. In the case of hydrophilic bacterial adhesion onto the substrate, the "brush effect" is enhanced with respect to water (Figure 2(C)). The methyl tilt angles decrease accordingly by $3.6^{\circ}$ and $4.4^{\circ}$ for $\mathrm{A}$ and B molecules in comparison to the ODT SAM conformation in water. On the contrary, the effect of hydrophobic bacteria is to decrease the $R$ value and so to flatten the ODT SAM: the methyl tilt angle increases of $3.3^{\circ}$ and $4.9^{\circ}$ for $A$ and B molecules with respect to water (Figure 2(D)).

These different behaviours can be rationalized by considerations of the hydrophobic/hydrophilic and hydrophobic/hydrophobic interactions [17]. The ODT SAM, composed of $\mathrm{CH}_{2}$ and $\mathrm{CH}_{3}$ groups, is a hydrophobic surface. When the ODT SAM is in contact with water molecules which are polar and hydrophilic, the system tends to remain separated in two phases, which is best achieved when the methyl 
terminal groups of the surface point up. This minimizes the interaction between the two phases, which maximizes the hydrogen bonding network inside the solvent and leads to a formation of an ordered water layer on the top of the ODT SAM.

In the case of hydrophobic Lactococcus lactis adhesion, only van der Waals interactions are present and there is no phase separation. Interactions are maximized when the ODT SAM is flattened because methylene group near the methyl terminal part can also be in interaction with bacteria and contributes to the attractive interactions. For hydrophilic bacteria adhesion, we expect a behaviour similar to water: it is indeed the case. Interestingly, the brush effect is larger for bacteria than for water. However, the complexity of the bacterial membrane is such that it is not possible to propose a simple explanation to this observation.

\section{Conclusion}

The present results demonstrate that the effect of bacterial adhesion can be observed by SFG spectroscopy and that bacteria alter the conformation of the surface, here an ODT SAM on a gold surface. We note the high sensitivity of SFG to molecular conformation: a small variation of the methyl tilt angle produces a measurable change of the SFG spectrum. The effect of water or bacteria consists of only a small adjustment of angles inside the SAM, which is found to depend on the hydrophobic or hydrophilic character of the medium interacting with the SAM. This demonstrates that the effect of bacterial adhesion depends primarily on the case of ODT SAM and Lactococcus bacteria, on bacterial physicochemical properties. It also implies that engineering a functionalized polymer or other surface layer to prevent the surface from being colonized [7, 8] must take into account the fact that bacteria might change the conformation of the protective layer. This phenomenon could explain the differences observed between predictive models and experimental adhesion measurements. In conclusion, this study shows that a general understanding of the interaction between bacteria and the surface at the molecular level is required to design new materials effective against bacterial contamination. The presence of other organic molecules such as proteins in the surrounding medium or in biofilms might also affect the conformation of the surface and it must be taken into account [18]. This is now under investigation.

\section{Acknowledgment}

The authors wish to thank the MIMA2 platform (INRA, Massy, France) for allowing us to use the scanning electron microscope and the CPBM platform of LUMAT (Orsay, France) for the use of their biological infrastructures.

\section{References}

[1] D. Lindsay and A. von Holy, "Bacterial biofilms within the clinical setting: what healthcare professionals should know," Journal of Hospital Infection, vol. 64, no. 4, pp. 313-325, 2006.

[2] A. C. L. Wong, "Biofilms in food processing environments," Journal of Dairy Science, vol. 81, no. 10, pp. 2765-2770, 1998.

[3] M. R. Nejadnik, H. C. van der Mei, W. Norde, and H. J. Busscher, "Bacterial adhesion and growth on a polymer brush-coating," Biomaterials, vol. 29, no. 30, pp. 4117-4121, 2008. 
[4] G. Speranza, G. Gottardi, C. Pederzolli et al., "Role of chemical interactions in bacterial adhesion to polymer surfaces," Biomaterials, vol. 25, no. 11, pp. 2029-2037, 2004.

[5] K. M. Wiencek and M. Fletcher, "Bacterial adhesion to hydroxyl- and methyl-terminated alkanethiol self- assembled monolayers," Journal of Bacteriology, vol. 177, no. 8, pp. 1959-1966, 1995.

[6] C. J. P. Boonaert, Y. F. Dufrêne, S. R. Derclaye, and P. G. Rouxhet, "Adhesion of Lactococcus lactis to model substrata: direct study of the interface," Colloids and Surfaces B, vol. 22, no. 3, pp. 171-182, 2001.

[7] G. Cheng, Z. Zhang, S. Chen, J. D. Bryers, and S. Jiang, "Inhibition of bacterial adhesion and biofilm formation on zwitterionic surfaces," Biomaterials, vol. 28, no. 29, pp. 4192-4199, 2007.

[8] P. M. Sivakumar, G. Iyer, L. Natesan, and M. Doble, "3'-Hydroxy-4-methoxychalcone as a potential antibacterial coating on polymeric biomaterials," Applied Surface Science, vol. 256, no. 20, pp. 6018-6024, 2010.

[9] T. S. Koffas, J. Kim, C. C. Lawrence, and G. A. Somorjai, "Detection of immobilized protein on latex microspheres by IR-visible sum frequency generation and scanning force microscopy," Langmuir, vol. 19, no. 9, pp. 3563-3566, 2003.

[10] J. Wang, S. M. Buck, and Z. Chen, "Sum frequency generation vibrational spectroscopy studies on protein adsorption," Journal of Physical Chemistry B, vol. 106, no. 44, pp. 11666-11672, 2002.

[11] C. Howell, M. O. Diesner, M. Grunze, and P. Koelsch, "Probing the extracellular matrix with sumfrequency-generation spectroscopy," Langmuir, vol. 24, no. 24, pp. 13819-13821, 2008.

[12] B. Bourguignon, W. Zheng, S. Carrez, A. Ouvrard, F. Fournier, and H. Dubost, "Deriving the complete molecular conformation of self-assembled alkanethiol molecules from sum-frequency generation vibrational spectra," Physical Review B, vol. 79, no. 12, Article ID 125433, 2009.

[13] E. Bulard, Z. Guo, W. Zheng et al., "Non-invasive vibrational SFG spectroscopy reveals that bacterial adhesion can alter the conformation of grafted"brush" chains on SAM," Langmuir, vol. 27, no. 8, pp. 4928-4935, 2011.

[14] M. P. Chapot-Chartier, E. Vinogradov, I. Sadovskaya et al., "Cell surface of Lactococcus lactis is covered by a protective polysaccharide pellicle," Journal of Biological Chemistry, vol. 285, no. 14, pp. 10464-10471, 2010.

[15] O. Habimana, C. Le Goff, V. Juillard et al., "Positive role of cell wall anchored proteinase PrtP in adhesion of lactococci," BMC Microbiology, vol. 7, article 36, 2007.

[16] B. Busson and A. Tadjeddine, "Non-uniqueness of parameters extracted from resonant secondorder nonlinear optical spectroscopies," Journal of Physical Chemistry C, vol. 113, no. 52, pp. 21895-21902, 2009.

[17] J.-C. Chottard, J. C. Depezay, and J. P. Leroux, Chimie Fondamentale, Études Biologiques et Médicales2: structure Moléculaire, Hermann, Paris, France, 1995.

[18] Y. H. An and R. J. Friedman, "Concise review of mechanisms of bacterial adhesion to biomaterial surfaces," Journal of Biomedical Materials Research, vol. 43, no. 3, pp. 338-348, 1998. 


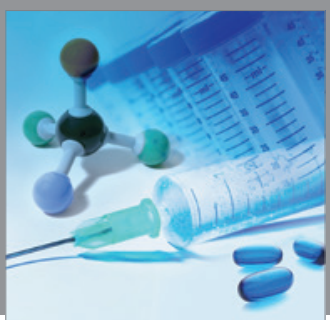

International Journal of

Medicinal Chemistry

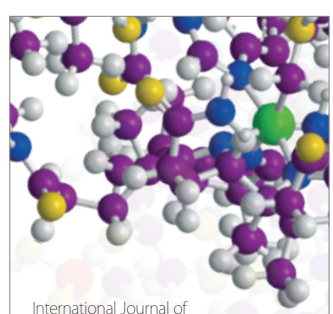

Carbohydrate Chemistry

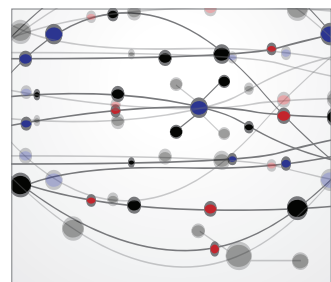

The Scientific World Journal
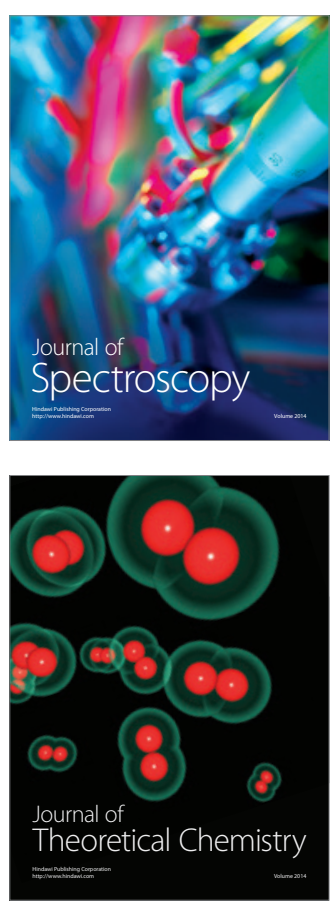
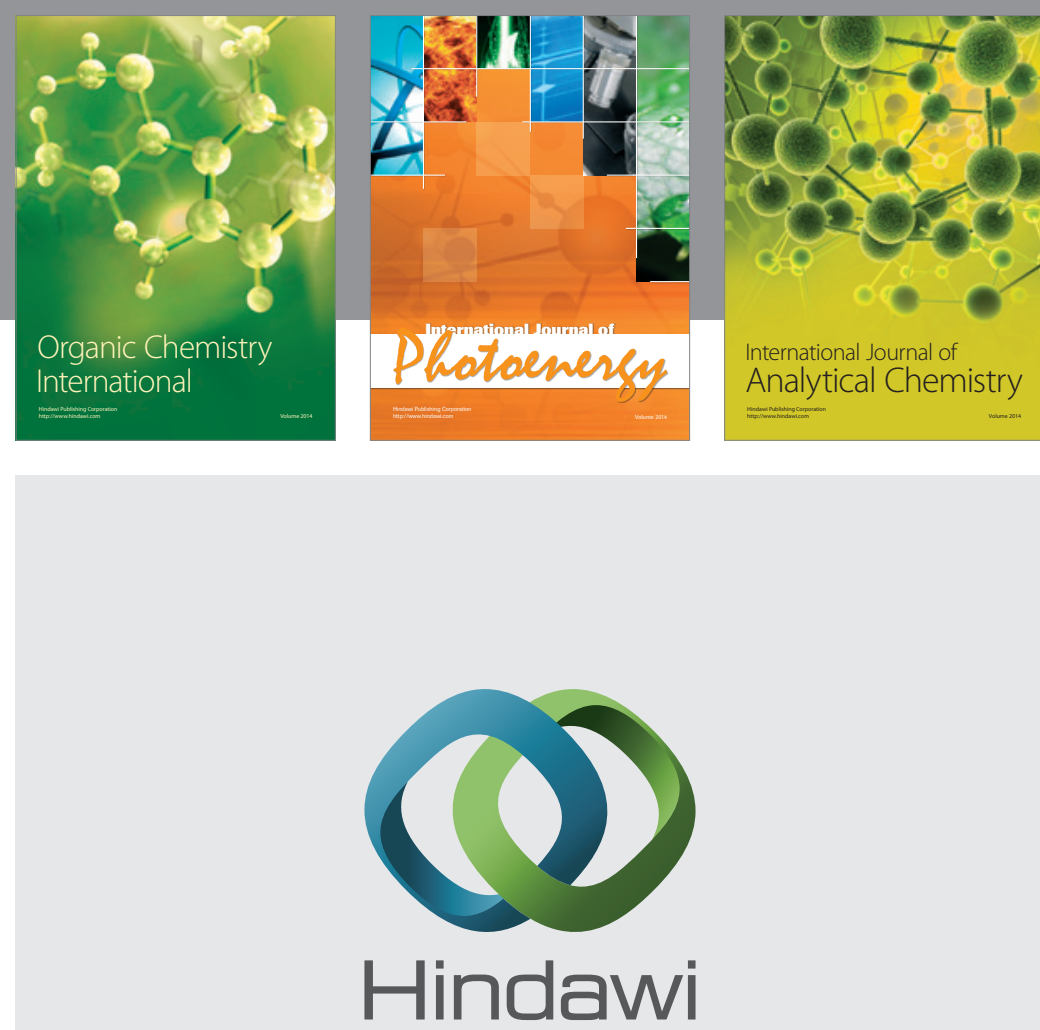

Submit your manuscripts at

http://www.hindawi.com
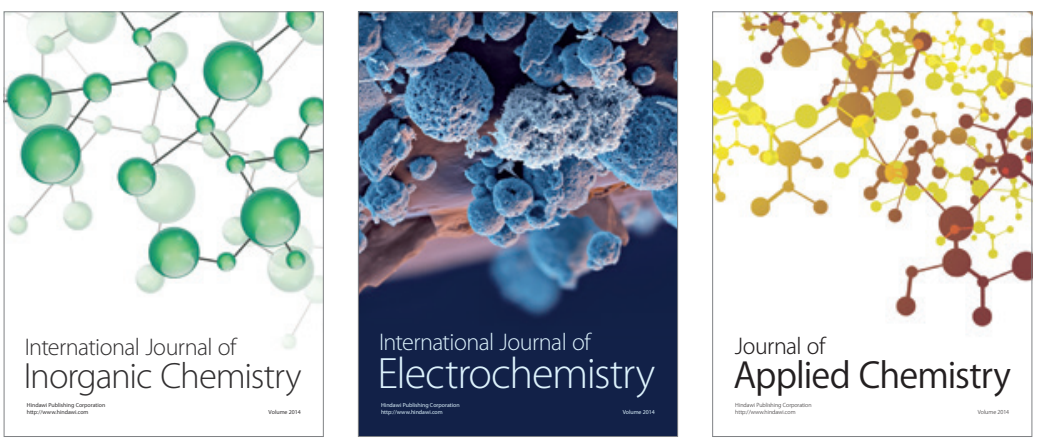

Journal of

Applied Chemistry
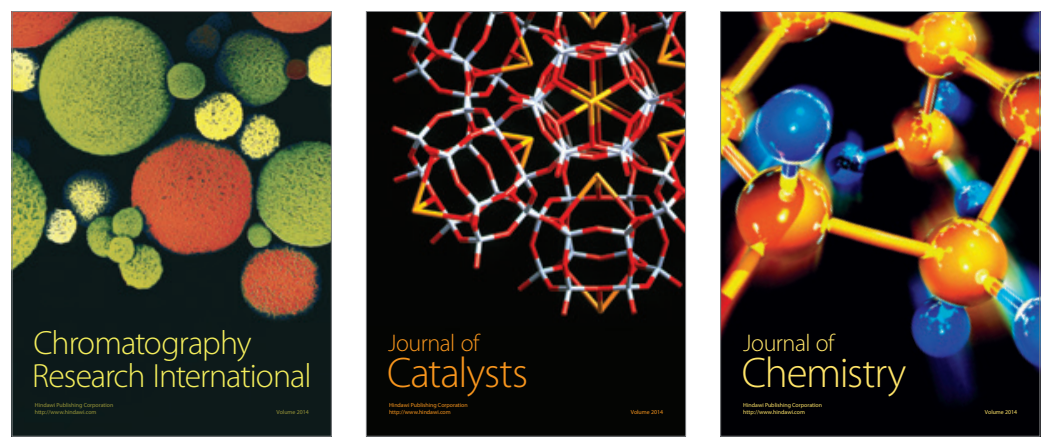
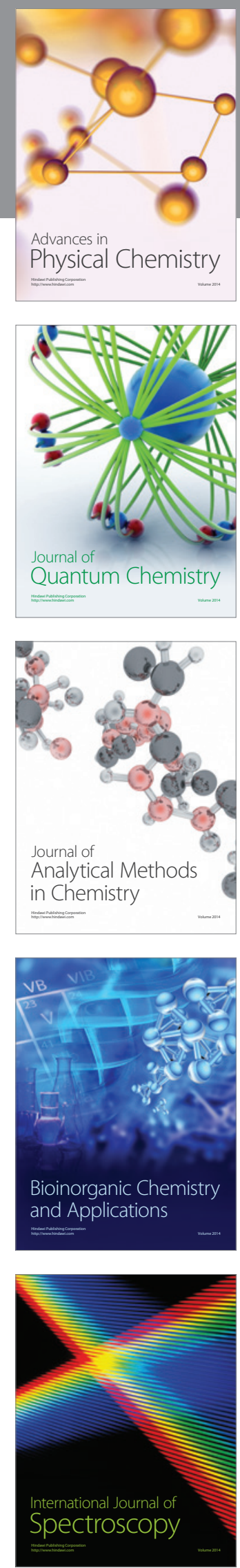